\title{
Non-Typhoidal Salmonella Isolated From Extraintestinal Specimens: Case Report
}

\author{
Sankarankutty Jaya ${ }^{1, *} ;$ Vipparti Harita $^{2}$ \\ ${ }^{1}$ Department of Microbiology, Shridevi Institute of Medical Sciences and Research Centre, Rajiv Gandhi University, Karnataka, India \\ ${ }^{2}$ Department of Microbiology and Serology, Narayana Hrudayalaya Hospital, Bangalore, India \\ *Corresponding author: Sankarankutty Jaya, Department of Microbiology, Shridevi Institute of Medical Sciences and Research Centre, Rajiv Gandhi University, Karnataka, India. Tel: \\ +91-9538896609, E-mail: drsjaya@gmail.com \\ Received: November 6, 2012; Revised: December 25, 2012; Accepted: February 12, 2013
}

Salmonella is a gram-negative, non-spore forming facultative anaerobic, motile bacilli. In developing countries, non-typhoidal Salmonella (NTS) accounts for the steadily increase in the proportion of human infections in recent decades. The clinical presentations of nontyphoidal Salmonella infection are protean, including gastroenteritis (the most common one), bacteremia, septic arthritis, osteomyelitis and endovascular infection.

Keywords: Synovial Fluid; Salmonella enterica; Suppuration

\section{Introduction}

Salmonella is a gram-negative, non-spore forming facultative anaerobic, motile bacilli. The genus Salmonella belongs to the family of Enterobacteriaceae and is named after Daniel E. Salmon who first isolated Salmonella cholerasuis from pigs in 1884 (1). The clinical presentations of non-typhoidal Salmonella infection are protean, including gastroenteritis (most common), bacteremia, septic arthritis, osteomyelitis and endovascular infection (2). Suppurated localized infections due to Salmonella spp. are secondary to bacteremia, after enteric fever or enterocolitis. This unusual clinical syndrome occurs in approximately1.5\% of non-typhoidal Salmonella infections (3). We report three cases of non-typhoidal Salmonella in pleural fluid, synovial fluid and pus: 1) pleural fluid in a patient with empyema and chronic renal failure for maintenance hemodialysis, 2) synovial fluid in a patient with pyogenic arthritis of knee and adrenal insufficiency syndrome, 3) Pus in a patient with osteosarcoma on chemotherapy.

\section{Case Presentation}

\subsection{Case 1}

This first subject was a 55-year-old man with a known case of grade 5 chronic kidney disease on maintenance hemodialysis presented with dyspnea and fever for one week. He was previously diagnosed to have chronic glo- merular nephritis, hypertension and anemia and previous admission for cardiac rhythm disorder. His pulse and blood pressure were normal and he was conscious, oriented and had decreased air entry on the right side. His TC (total capacity) was $22400 / \mathrm{cu} \mathrm{mm}$, neutrophils at $90.2 \%$, serum creatinine of $6 \mathrm{mg} / \mathrm{dL}$ and blood urea of $37 \mathrm{mg} / \mathrm{dL}$. Chest X-ray showed moderate to large pleural effusion with consolidation. The CT scan showed large loculated pleural effusion with pleural thickening suggestive of empyema and collapse consolidation of the right lower and middle lobe (Figure 1). Pleural fluid was aspirated and sent for cytology and culture. Cytological analysis showed that fluid was turbid with a cell count of $34000 / \mathrm{cu} \mathrm{mm}$, neutrophils at $80 \%$ and lymphocytes at $20 \%$. Thoracoscopy was performed during the following day and they noticed adhesions. Implantable cardioverter defibrillator, ICD was placed. Microbiologically, there were grayish white non lytic colonies on blood agar and non-lactose fermenting colonies on MacConkey agar (Figure 2).

Cells were slender gram-negative bacilli, catalase positive, oxidase negative and motile. Biochemical reactions showed that the bacteria fermented mannitol and they were motile. TSI showed alkaline/acid with abundant $\mathrm{H}_{2} \mathrm{~S}$ production, citrate utilization, urea not hydrolysed, identified as Salmonella group by Vitek 2 compact which was later sent for serotyping. It was positive for antisera A-G, factor 4 and Factor iH. Hence, the bacteria was Salmonella typhimurium.

Implication for health policy/practice/research/medical education: The study is conducted for the purpose of medical education.

Copyright (c) 2013, Infectious Diseases and Tropical Medicine Research Center. This is an open-access article distributed under the terms of the Creative Commons Attribution License, which permits unrestricted use, distribution, and reproduction in any medium, provided the original work is properly cited. 


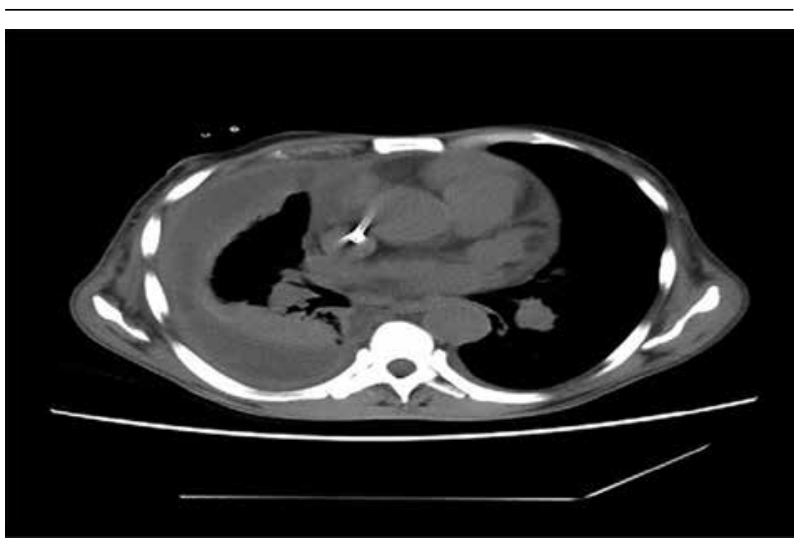

Figure 1. CT Scan of Pleural Effusion

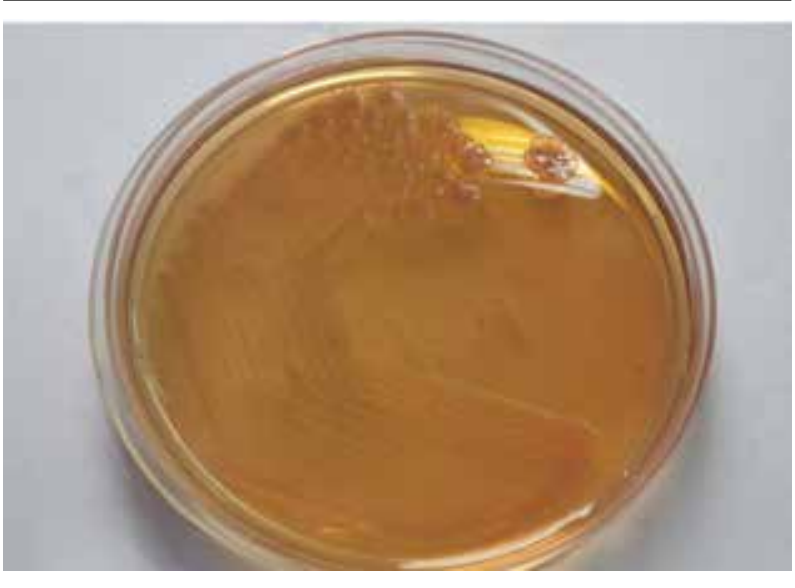

Figure 2. Non-lactose Fermenting Colony on MacConkey Agar

It was sensitive to ampicillin, ceftriaxone, cefoperazone, cefepime, fluoroquinolones, chloramphenicol and carbapenems. Blood cultures from the same patient was negative for growth. He was administered cefoperazone/ sulbactam. The drain reduced from $250 \mathrm{~mL}$ to $160 \mathrm{~mL}$ gradually and improved symptomatically. The patient was discharged after two weeks with ICD (implantable cardioverter defibrillator). After one week, the patient was brought back to the hospital with history unconsciousness during dialysis. On examination, he did not respond to commands but showed response to pain. With ICD (implantable cardioverter defibrillator) in place, he was immediately intubated. Salmonella group sensitive to ampicillin, ceftriaxone, cefoperazone, cefepime, fluoroquinolones, chloramphenicol and carbapenems grew in pleural fluid culture. On the same day, he was discharged against medical advice.

\subsection{Case 2}

A 32-year-old man from Tumkur was admitted to the hospital with a one week history of right knee joint pain associated with fever and chills. He had a history of central hypothyroidism and adrenal insufficiency on treatment with steroids. He had a known case of arthritis. On examination, he had knee joint swelling, tenderness and local rise of temperature suggestive of pyogenic arthritis. Laboratory investigation showed total cholesterol (TC) 22.1, 80.5\% neutrophils, ESR 56 mm/L. Other biochemistry parameters were normal. Fluid was aspirated and sent for cytology, culture and sensitivity. Microscopic examination revealed a slightly turbid fluid and sheets of polymorphs. Microbiologically - there were grayish white non-lytic colonies on blood agar and non-lactose fermenting colonies on MacConkey agar. Cells were slender gram-negative bacilli, catalase positive, oxidase negative and motile. Biochemical reactions showed that the bacteria fermented mannitol and they were motile. Triple sugar iron (TSI) showed alkaline/acid with abundant $\mathrm{H} 2 \mathrm{~S}$ production, citrate utilization, Urea not hydrolysed, Identified as Salmonella group by Vitek 2 compact, which was later sent for serotyping. The result was positive for antisera A-G, factor 4 and factor $\mathrm{iH}$. Hence, it the bacteria were Salmonella typhimurium. They were sensitive to ampicillin, ceftriaxone, cefoperazone, cefepime, fluoroquinolones, chloramphenicol and carbapenems. The patient was treated with sensitive antibiotics and then discharged.

\subsection{Case 3}

A 22-year-old female from an Arab country referred to our hospital with a huge swelling of the left thigh (partly amputed) and difficulty in breathing. On examination, there was a huge soft tissue tumor. Her chest X-ray showed pneumothorax for which ICD was inserted. She was a case of malignant osteosarcoma on chemotherapy. She had undergone disarticulation of the left hip joint in her country due to diagnosed osteosarcoma of femur two months prior to her admission to our hospital. She developed infection at this amputation site which had grown Klebsiella spp earlier. She was surgically treated in our hospital with amputation of the stump considering the spread of sarcoma and infection. She developed pus at the amputation site after a few days, which was sent for culture and sensitivity. The culture grew extended-spectrum $\beta$-lactamase (ESBL) Klebsiella pneumonia (sensitive to carbapenems, aminoglycosides and tigecycline) and multidrug-resistant Pseudomonas aeruginosa (sensitive to colistin) (identification and susceptibility by Vitek 2 ). After a few days, lumbar drain tip was placed surgically and pus was sent for culture, which grew Salmonella enterica spp enteric sensitive to carbapenems, piperacillin-tazobactam and tigecycline, and resistant to fluoroquinolones, ESBL Klebsiella pneumonia and multidrug-resistant Pseudomonas aeruginosa. Repeated culture from the wound was sent to the laboratory, which grew the same organisms. The Salmonella strain was sent 
for serotyping, which was reported as biochemically suggestive of Salmonella typhimurium. It was negative for antisera A-G, factor 4 and Factor iH and Salmonella species biochemically resembling Salmonella typhimurium.

\section{Conclusions}

In developing countries, non-typhoid Salmonella (NTS) accounts for the steadily increase in the proportion of human infections during recent decades (4). The most common clinical presentation of Salmonella infection is acute enterocolitis, but Salmonella can cause fever, sustained bacteremia, and even extraintestinal infectious complications like pneumonia without gastrointestinal manifestations (5). In the absence of concurrent pulmonary infection, a pleural empyema caused by nontyphoidal Salmonella is rare, with only 16 cases reported to date (6). In industrialized countries, most individuals infected with non-typhoidal salmonella (NTS), experience mild gastrointestinal illnesses involving diarrhea, chills, abdominal cramps, fever, head and body aches, nausea and vomiting. Infections are acquired through food poisoning and are usually self-limiting and antimicrobial treatment is not recommended for uncomplicated illnesses. In most cases, outbreaks of NTS infection are caused by Salmonella enteric serotype Typhimurium ( $S$. Typhimurium) and Salmonella enterica serotype Enteritidis (S. Enteritidis). A variety of food have been implicated as vehicles transmitting salmonellosis to humans, including poultry, beef, pork, eggs, milk, cheese, fish, shellfish, fresh fruit and juice and vegetables (7).

Localization of infection may occur at any site after Salmonella bacteremia, irrespective of the associated clinical syndrome. As might be anticipated, localization at distant sites occurs relatively frequently in patients with the Salmonella bacteremia syndrome but rarely in patients with enterocolitis. Localized infection has been reported for the thyroid, meninges, bone, heart, lungs, adrenals, pancreas, spleen, liver, testis, pericardium, soft tissues, areas of necrosis or infarction, benign or malignant tumors and cysts (8). Ramos et al. reported that the most frequent site of non-digestive infection was the urinary tract (30\%), followed by the respiratory tract (20\%) and the osteoarticular system (19\%)(9). Various pathogenic mechanisms have been proposed, i.e. extension from a nearby pulmonary site or hematogenous dissemination to the lung or pleura (3). In a review of extraintestinal infections caused by non-typhi Salmonella serotypes over a nine-year period, Ruiz et al. concluded that the greatest risk of acquiring local infection is associated with the existence of underlying illness or immunosuppression. During the period analyzed, they studied 1543 patients from whom some serotype of non-typhoid Salmonella was isolated. Extra-intestinal locations were involved in only $1.8 \%$ of the patients and most cases occurred in children or elderly patients. In only one case Salmonella typhimurium was isolated from pleural effusion (3). Another case of empyema with Salmonella paratyphi B was reported by Annamalai et al. (10).

Localized infection can involve any site of the body including bones and joints. Majority of infections occur in patients with pre-existing disease such as haemoglobinopathy, arthritis including rheumatoid arthritis, osteoarthritis, gout, previous trauma, surgery and immunosupression (11). Of the bacterial pathogens, Salmonella arthritis occurs very infrequently, accounting for only about $1 \%$ of all cases and even that usually represents metastatic extension of non-typhoidal Salmonella. Septic arthritis is an extremely rare complication of typhoidal Salmonella (12). Agnihotri et al. reported a case of hip arthritis with Salmonella typhi (11). Similarly Mahajan et al. reported four cases of septic arthritis with Salmonella typhi (13). Govender et al. reported 16 cases of septic arthritis with Salmonella typhimurium (14). Salmonella was isolated in pus in a few cases, Sengupta et al. reported a case of Salmonella typi in post-op wound infection (15) and Abhijit et al. reported a case of Salmonella enteritidis from pus (16). An extra-intestinal focus of infection was noted in one third of patients, most commonly involving the lung and soft tissue. Patients with severe clinical immunosuppression had higher mortality, were presented more commonly with primary bacteraemia, leukopenia and opportunistic infections and absence of gastroenteritis (17). However, Najmuddin reported three cases of nontyphoidal salmonellosis in immunocompetent patients presenting over a one year period (18). Extra intestinal manifestation of non-typhoidal Salmonella infections such as empyema, pyogenic arthritis and surgical site infections are rare and seen in immunocompramised patients. Hence high degree of suspicion and appropriate antibiotics has to be administered for proper treatment.

\section{Acknowledgements}

We would like to thank the Department of Microbiology, St. Johns Hospital Bangalore for helping us with the serotyping.

\section{Authors' Contribution}

Both authors collaborated equally.

\section{Financial Disclosure}

The authors declare that there are no conflicts of interest.

\section{Funding/Support}

The study did not receive any financial support.

\section{References}

1. Miller SI, Pegues DA. Salmonella species including typhoid fever. In: Mandell GL, Bennett JE, Dolin R, editors. Principles and prac- 
tice of infectious diseases. New York, NY, USA: Churchill Livingstone; 2000. p. 2344-63.

2. Hohmann EL. Nontyphoidal salmonellosis. Clin Infect Dis. 2001;32(2):263-9.

3. de Lope ML, Batalha P, Sosa M, Rodriguez-Gomez FI, Sanchez-Munoz A, Pujol E, et al. Pleural empyema due to Salmonella enteritidis in a non-immunocompromised patient. EurJ Clin Microbiol Infect Dis. 2004;23(10):792-3.

4. Yen YF, Wang FD, Chiou CS, Chen YY, Lin ML, Chen TL, et al. Prognostic factors and clinical features of non-typhoid Salmonella bacteremia in adults. J Chin Med Assoc. 2009;72(8):408-13.

5. Afridi FI, Farooqi BJ, Hussain A. Pleural empyema due to Salmonella typhi.J Coll Physicians Surg Pak. 2012;22(12):803-5.

6. Kam JC, Abdul-Jawad S, Modi C, Abdeen Y, Asslo F, Doraiswamy V, et al. Pleural Empyema due to Group D Salmonella. Case Rep Gastrointest Med. 2012;2012:524561.

7. Kariuki S, Revathi G, Kariuki N, Kiiru J, Mwituria J, Muyodi J, et al. Invasive multidrug-resistant non-typhoidal Salmonella infections in Africa: zoonotic or anthroponotic transmission? J Med Microbiol. 2006;55(Pt5):585-91.

8. Rim MS, Park CM, Ko KH, Lim SC, Park KO. Pleural empyema due to Salmonella: a case report. Korean J Intern Med. 2000;15(2):138-41.

9. Ramos JM, García-Corbeira P, Aguado JM, Alés JM, Soriano F. Classifying extraintestinal non-typhoid Salmonella infections. QJM. 1996;89(2):123-6.
10. Annamalai A, Shreekumar S, Muthukumaran R. Empyema in enteric fever due to Salmonella parathyphi B. Dis Chest. 1969;55(1):73-4.

11. Agnihotri N, Dhingra MS, Gautam V, Gupta V, Kaushal R, Mehta D. Salmonella typhi septic arthritis of hip-a case report.Jpn J Infect Dis. 2005;58(1):29-30

12. Chiu S, Chiu CH, Lin TY, Luo CC, Jaing TH. Septic arthritis of the hip caused by Salmonella typhi. Ann Trop Paediatr. 2001;21(1):88-90.

13. Mahajan RK, Chaudhary J, Chaskar P, Arya RK, Duggal N, Hans C Septic arthritis due to Salmonella Typhi in children-A case series. The Journal of Bioscience and Medicine. 2012;2(2).

14. Govender S, Chotai PR. Salmonella osteitis and septic arthritis. J Bone Joint Surg Br.1990;72(3):504-6.

15. Sengupta S, Jagadishchandra K, Murty R, Shivananda PG. An unusual post-operative wound infection with Salmonella typhi: case report. Indian J Med Sci. 2000;54(4):149-50.

16. Abhijit AA, SuNitA N. The Study of salmonellosis with reference to Salmonella typhi in enteric fever patients. J Clin Diagn Res. 2011;5(3):467-9.

17. Dhanoa A, Fatt QK. Non-typhoidal Salmonella bacteraemia: epidemiology, clinical characteristics and its' association with severe immunosuppression. Ann Clin Microbiol Antimicrob. 2009;8:15.

18. Najmuddin A, Khan S, Hussain K. Spectrum of nontyphoidal Salmonella infections among immunocompetent individuals. Chest. 2012;142. 